\title{
Differentially expressed circular RNAs in a murine asthma model
}

\author{
HUI BAO ${ }^{1 *}$, QIUYAN ZHOU ${ }^{1 *}$, QIUJU LI $^{1}$, MENGMENG NIU $^{1}$, SANFENG CHEN $^{2}$, \\ PINGCHANG YANG ${ }^{1}$, ZHIGANG LIU $^{1}$ and LIXIN XIA ${ }^{1}$ \\ ${ }^{1}$ Research Center of Allergy and Immunology, Shenzhen University School of Medicine, Shenzhen, Guangdong 518060; \\ ${ }^{2}$ Department of Internal Medicine, Aged Care Hospital of Hangzhou, Hangzhou, Zhejiang 310015, P.R. China
}

Received April 23, 2020; Accepted September 25, 2020

DOI: $10.3892 / \mathrm{mmr} .2020 .11617$

\begin{abstract}
Allergic asthma is one of the most common allergic diseases; however, the mechanisms underlying its development have yet to be fully elucidated. Although allergic diseases are inheritable, genetic variance alone cannot explain the notable increase in the prevalence of allergic diseases over a short period of time in recent decades. Recently, research focus has been shifting to epigenetic factors, such as non-coding RNAs. Circular RNAs (circRNAs) are involved in the pathogenesis of various diseases. The aim of the present study was to further elucidate the etiology of allergic asthma by analyzing aberrantly expressed circRNAs in a murine asthma model. A mouse model of house dust mite allergen-induced asthma was established, and the qualified libraries were sequenced using next-generation sequencing. The expression levels of circRNAs were validated by reverse transcription-quantitative PCR (RT-qPCR) analysis. Gene Ontology (GO) and Kyoto Encyclopedia of Genes and Genomes (KEGG) pathway analyses were performed for biological pathway classification and enrichment analysis of the aberrantly expressed circRNAs. In addition, the interaction network of the differentially expressed circRNAs and microRNAs (miRNAs) was constructed using Cytoscape. By next-generation sequencing, a total of 150 circRNAs were revealed to be upregulated and 130 were downregulated in the murine asthma model group compared with in the control group. GO and KEGG analyses demonstrated that the differentially expressed circRNAs were mainly involved in processes such as 'autoimmune disease', 'cell adhesion molecules (CAMs)' and 'endocytosis', among others. The expression levels of six circRNAs, namely three upregulated (circ_0000909, circ_0000629 and circ_0000455) and three downregulated (circ_0001454, circ_0000723 and
\end{abstract}

Correspondence to: Professor Lixin Xia, Research Center of Allergy and Immunology, Shenzhen University School of Medicine, 3688 Nanhai Road, Shenzhen, Guangdong 518060, P.R. China E-mail: xialixin@126.com

*Contributed equally

Key words: asthma, allergy, house dust mites, circular RNAs, expression profile circ_0001389) circRNAs, were validated by RT-qPCR. In conclusion, the analyses suggested that circRNAs performed critical functions via endocytosis (such as macrophage endocytosis), cell adhesion molecules and lipid metabolism in allergic asthma. The interaction network revealed that certain miRNAs that may serve a role in asthma could be regulated by the differentially expressed circRNAs.

\section{Introduction}

Allergic diseases, including asthma, affect $30-40 \%$ of the global population (1). The symptoms of allergic diseases range from local mild reactions to severe anaphylaxis. A polarized T helper type $2(\mathrm{Th} 2)$ immune response and degranulation of mast cells are characteristic in allergic diseases $(2,3)$. Despite the fact that the exact mechanisms underlying these diseases have been extensively investigated, they remain to be fully elucidated. Although allergic diseases, such as asthma, are inheritable (4), accumulating evidence has indicated that epigenetic factors, such as non-coding RNAs [e.g., microRNAs (miRNAs/miRs), long non-coding RNAs (lncRNAs) or circular RNAs (circRNAs)] may have a role in these diseases $(5,6)$. Alterations in the expression of several miRNAs have been reported to be associated with the development of allergic diseases. In allergic inflammation, miR-155 was revealed to be significantly upregulated $(7,8)$; it targeted the cytotoxic T-lymphocyte-associated protein 4 mRNA in patients with atopic dermatitis and protected group 2 innate lymphoid cells from apoptosis, thereby promoting type 2 immunity. Furthermore, inhibition of miR-155 resulted in dampened expression of the Th2 cytokines, interleukin (IL)-5 and IL-13 (9). Abnormal lncRNA expression has also been observed in allergies. Zhang et al (10) profiled human $\mathrm{CD} 4^{+} \mathrm{T}$-cell subsets and identified a Th2-specific IncRNA, GATA3-AS1. Notably, peripheral blood mononuclear cells and $\mathrm{CD} 4^{+} \mathrm{T}$-cell clones stimulated by an allergic challenge displayed increased expression levels of GATA3-AS1.

circRNAs are single-stranded, highly conserved RNAs that take the form of a covalently closed continuous loop (11). circRNAs are involved in the pathogenesis of various diseases (12). Notably, circRNAs have been reported to act as miRNA sponges through multiple miRNA response elements, modulating the expression of parental genes (13). For example, circHIPK 3 has been shown to bind to miR-558, resulting in upregulation of its target protein heparanase, thus suppressing 
metastasis and angiogenesis of bladder cancer (14). Recently, certain circRNAs have been demonstrated to encode peptides (15). However, the functions of circRNAs in allergic diseases remain unclear. In the present study, the expression levels of aberrantly expressed circRNAs were profiled in a murine asthma model and their possible contributions were investigated, with the aim of providing novel insights into the etiology and pathogenesis of asthma.

\section{Materials and methods}

Mice. A total of six female BALB/c mice (age, 6-8 weeks; weight, $33 \pm 0.2 \mathrm{~g}$ ) were purchased from Guangdong Medical Laboratory Animal Center and maintained under specific pathogen-free conditions in the animal facility of Shenzhen University (Shenzhen, China). A total of five mice were housed per cage in a laminar air flow cabinet, maintained at $23 \pm 2^{\circ} \mathrm{C}$ at a relative humidity of $55 \pm 5 \%$ with a $12 \mathrm{~h}$ dark/light cycle. All mice received a standard diet and water ad libitum. The experimental protocols of the present study were reviewed and approved by the Animal Care and Use Committee of Shenzhen University. The mice were divided into control $(n=3$, namely $\mathrm{N} 1, \mathrm{~N} 2$ and $\mathrm{N} 3)$ and asthma model groups $(\mathrm{n}=3$, namely M1, M2 and M3).

Development of a murine asthma model. A house dust mite (HDM) allergen-induced asthma mouse model was established, as previously described (16), the method of mouse nose drops was changed to subcutaneous injection for the consistency of the sensitization process. Female $\mathrm{BALB} / \mathrm{c}$ mice were sensitized subcutaneously with $20 \mu \mathrm{g}$ crude Dermatophagoides farinae (HDM) extract (Greer Laboratories, Inc.) in $100 \mu 1$ saline on days 0,7 and 14 , and were challenged intranasally with $20 \mu \mathrm{g}$ HDM in $50 \mu \mathrm{l}$ saline for 5 consecutive days from day 20 onwards. The control group only received treatment with PBS. Airway hyperresponsiveness (AHR) to inhaled methacholine was analyzed to evaluate changes in airway resistance in response to allergen exposure and challenge. On day 26 , the mice were sacrificed using $\mathrm{CO}_{2}$ at flow rate of $20 \%$ volume/min (17), and murine lung tissues were dissected and analyzed for inflammation. Mice lung tissues were dissected and RNA was isolated for circRNA profiling. Bronchoalveolar lavage fluid (BALF) was harvested via lung tissue lavage with ice-cold PBS. The methods for differentiating BALF cells were previously described in detail (18). Sections from mouse lungs were fixed at room temperature for 12 min with $4 \%$ neutral buffered formalin, and were then stained with hematoxylin and eosin and periodic acid-Schiff. Blood was collected to screen for serum antibodies against HDM allergens.

Histopathological examination of lung tissue. The right middle lung of mice was harvested and fixed in $4 \%$ paraformaldehyde at room temperature for $24 \mathrm{~h}$. After dehydration and paraffin embedding, glass slides were coated with $4-\mu \mathrm{m}$ sections. Subsequently, the sections were stained with hematoxylin and eosin (cat. no. G1005; Servicebio, Inc.) for $5 \mathrm{~min}$ at room temperature and with periodic acid-Schiff reagent (cat. no. G1008; Servicebio, Inc.) for $10 \mathrm{~min}$ at room temperature. The lung tissue sections were examined at x200 magnification using an optical microscope (DS-Ri2; Nikon Corporation).

Measurement of serum levels of HDM-specific IgE and IgGI. Blood samples (100 $\mu \mathrm{l}$ per mouse) from the retro orbital venous plexus were collected using a heparinized capillary after the mice were anesthetized by isoflurane (RWD Life Science), and stored at room temperature for $2 \mathrm{~h}$. The samples were centrifuged $\left(3,000 \mathrm{x} \mathrm{g}, 10 \mathrm{~min}, 4^{\circ} \mathrm{C}\right)$ to isolate the serum and stored at $-80^{\circ} \mathrm{C}$ until further analysis. The serum levels of HDM-specific IgE and IgG1 were assayed using an ELISA kit (cat. no. DY008; R\&D Systems, Inc.) according to the manufacturer's protocol. The 96-well ELISA plates were coated overnight at $4^{\circ} \mathrm{C}$ with $100 \mu \mathrm{l}$ of a $4 \mu \mathrm{g} / \mathrm{ml}$ solution of HDM in carbonate-bicarbonate buffer ( $\mathrm{pH}$ 9.6; Sigma-Aldrich; Merck KGaA). ELISA was performed using the dilutions of each serum samples, and the dilutions used were 1/4 for sIgE and 1/500 for sIgG1. ELISA was prepared using the Reagent diluent (cat. no. DY995; R\&D Systems, Inc.) and Goat Anti-Mouse IgG1-horseradish peroxidase (HRP; 1:2,000; cat. no. 1071-05; Southern Biotech) or Goat Anti-Mouse IgE-HRP (1:2,000; cat. no. 1110-05; Southern Biotech). The other steps were performed following the manufacturer's instructions.

Flow cytometry. For detection of eosinophils, red blood cells were lysed using ammonium chloride lysis buffer (Sigma-Aldrich; Merck KGaA). Absolute counting beads (cat. no. C36950; Invitrogen; Thermo Fisher Scientific) were mixed with the BALF cells and assessed via flow cytometry. The numbers of BALF cells were calculated by comparing the ratio of beads events to cell events according to the manufacturer's protocol. The BALF cells were stained with antibodies against CD3-FITC (cat. no. 100203; Biolegend, Inc.), B220-FITC (cat. no. 103205, Biolegend, Inc.), CCR3-PE (cat. no. 144505; Biolegend, Inc.), CD11c-PerCP/Cyanine5.5 (cat. no. 117327; Biolegend, Inc.) and MHCII-APC (cat. no. 116417; BD Biosciences) for $30 \mathrm{~min}$ at $4^{\circ} \mathrm{C}$. The cells were stained for neutrophils, mononuclear cells and lymphocytes (data not shown), and eosinophils using a CytoFLEX flow cytometer (Beckman Coulter, Inc.), and data collected were analyzed with FlowJo V10 software (FlowJo, LLC). Granulocytes were recognized as non-autofluorescent highly granular (SSC ${ }^{\text {hi }}$ ) cells, and within this gate, eosinophils were defined as cells expressing the eotaxin receptor CCR3 and with low expression of MHCII, B220 and CD3. Neutrophils had a similar scatter profile as eosinophils but lacked CCR3 expression. Lymphocytes were identified as $\mathrm{FSC}^{\mathrm{lo}} / \mathrm{SSC}^{\mathrm{lo}}$ and expressing CD3 or B220. Mononuclear cells expressed high levels of MHCII and CD11c.

Non-invasive measurement of airway function. Airway responsiveness was measured $24 \mathrm{~h}$ after the final exposure to HDM extract by recording respiratory pressure curves using barometric unrestrained whole-body plethysmography (Buxco; emka TECHNOLOGIES S.A.S) in response to inhaled methacholine (acetyl- $\beta$-methylcholine chloride; Sigma-Aldrich; Merck KGaA) in conscious unrestrained mice. Airway responsiveness was expressed as enhanced pause (Penh), as described previously (19). Briefly, mice were placed 
in a whole-body chamber and basal readings were obtained and averaged for a 3-min period. Subsequently, increasing doses of methacholine $(0-100 \mathrm{mg} / \mathrm{ml})$ were aerosolized for $3 \mathrm{~min}$, and readings were taken and averaged for $3 \mathrm{~min}$ after each nebulization.

RNA extraction and library construction. Total RNA was extracted from the tissues using TRIzol ${ }^{\circledR}$ reagent (Invitrogen; Thermo Fisher Scientific, Inc.), according to the manufacturer's instructions. Total RNA was qualified and quantified using a NanoDrop (NanoDrop; Thermo Fisher Scientific, Inc.) and Agilent 2100 bioanalyzer (Agilent Technologies, Inc.). Subsequently, total RNA was treated with DNase I, which degraded double-stranded and single-stranded DNA. Ribosomal RNA was removed using the Ribo-off rRNA Depletion kit (Vazyme Biotech Co., Ltd.) and linear RNA was removed using RNase R (Epicentre; Illumina, Inc.). Purification was performed using Agencourt RNAClean XP magnetic beads (Invitrogen; Thermo Fisher Scientific, Inc.). All steps were performed according to the manufacturer's protocols. The distribution of the fragment sizes was determined using the Agilent 2100 bioanalyzer and quantitated using a BMG microplate reader (Omega Bio-Tek, Inc.). Finally, the libraries were sequenced on the BGISEQ-500 (BGI Group). Raw reads were filtered out for low quality, linker contamination and excessively high levels of unknown base N (SOAPnuke software v1.5.2; https://github.com/BGI-flexlab/SOAPnuke), and then clean reads were aligned to the reference genome (Mus_musculus, UCSC_mm9; ftp://hgdownload.soe.ucsc. edu/goldenPath/mm9/).

Differentially expressed circRNAs. circRNAs were detected and identified using CIRI $(20,21)$ and Find_circ $(22,23)$, and the results of the two types of software were integrated based on the circRNA start and stop positions (combining circRNAs with start and stop positions within the first and last 10 bases into one class). The expression of circRNAs was calculated based on the number of junction reads aligned to the two ends of the circRNA. Since two softwares, CIRI (BWA-MEM genome alignment algorithm) and Find_circ (Bowtie2 genome alignment algorithm), were used in the profiling, the final numbers of junction reads were the mean of the two results. Junction reads per billion mapped reads was used to homogenize each sample, and the EBseq (24) algorithm was used to detect differentially expressed circRNAs between control and model groups. The circRNAs included in circBase (25) were provided with corresponding ID numbers in the present study. circRNAs exhibiting fold changes $\geq 2.0$ with P-values $\leq 0.05$ were classified as significantly differentially expressed circRNAs. The differentially expressed genes (DEGs) obtained from all groups were analyzed by bidirectional clustering using the Pheatmap package (v1.0.12; rdocumentation.org/packages/pheatmap/versions/1.0.12) and presented as heatmaps.

Validation of differentially expressed circRNAs and mRNAs. Total RNA from lung tissues was extracted using TRIzol ${ }^{\circledR}$ reagent. Total RNA $(1 \mu \mathrm{g})$ was treated with RNase $\mathrm{R}$ for circRNA RT-qPCR, or without RNase R for mRNA RT-qPCR, and first-strand cDNA was synthesized using the First Strand
cDNA Synthesis kit for reverse transcription-quantitative PCR (RT-qPCR) analysis (Invitrogen; Thermo Fisher Scientific, Inc.), according to the manufacturer's instructions. First-strand cDNA was synthesized using $8 \mu \mathrm{l}$ First Strand Synthesis Act D Mix and SuperScript II Reverse Transcriptase at $40^{\circ} \mathrm{C}$ for $1 \mathrm{~h}, 90^{\circ} \mathrm{C}$ for $5 \mathrm{~min}$ and $0^{\circ} \mathrm{C}$ for $5 \mathrm{~min}$. qPCR was performed on an ABI ViiA7 real-time PCR detection system (Applied Biosystems; Thermo Fisher Scientific, Inc.) using QuantiFast SYBR Green PCR kit (Qiagen, Inc.). The PCR mixture was incubated under the following conditions: One cycle at $95^{\circ} \mathrm{C}$ for $2 \mathrm{~min}$, followed by 40 cycles at $94^{\circ} \mathrm{C}$ for $10 \mathrm{sec}, 60^{\circ} \mathrm{C}$ for $10 \mathrm{sec}$ and $72^{\circ} \mathrm{C}$ for $40 \mathrm{sec}$, and a final extension step of $72^{\circ} \mathrm{C}$ for $5 \mathrm{~min}$. Data were analyzed using the $2^{-\Delta \Delta \mathrm{Cq}}$ method (26) and expressed as fold change relative to untreated controls. The circRNA levels were normalized to the internal control gene GAPDH. Differentially expressed circRNAs were identified by unpaired two-tailed Student's t-test between two groups and fold change between two samples. All the reactions were assessed with three biological replicates. The primers were designed by ABI Primer Express Software v2.0 (Applied Biosystems; Thermo Fisher Scientific, Inc.) and synthesized by BGI Group. The primer sequences used in the present study are listed in Table I.

Gene Ontology (GO) and Kyoto Encyclopedia of Genes and Genomes (KEGG) pathway analyses. GO was divided into three major functional categories: Molecular function, cellular component and biological process. According to the results of the DEGs, the clusterProfiler package (version 3.5.0; bioconductor.org/packages/release/bioc/html/clusterProfiler.html) in $\mathrm{R}$ software (version 3.6.0; cran.r-project.org) was used for GO and KEGG pathway enrichment analysis Subsequently, the P-value underwent false discovery rate (FDR) correction for $\mathrm{q}$-value, and the pathways with $\mathrm{q} \leq 0.01$ were considered as being significantly enriched. The directed acyclic graph (DAG) was a graphical display of the $\mathrm{GO}$ enrichment analysis results of genes from different circRNA sources.

Construction of a circRNA regulatory network. The targeted associations between mouse miRNAs and circRNAs were predicted using the miRanda (27) algorithm. The circRNA-miRNA interaction network was visualized using Cytoscape 3.7.1 software (28).

Statistical analysis. Data are expressed as mean \pm SEM. Statistical significance for normally distributed samples was assessed using an independent two-tailed Student's t-test or one-way ANOVA followed by the Bonferroni correction for multiple comparisons. All analyses were performed with GraphPad Prism 7 software (GraphPad Software, Inc.). $\mathrm{P} \leq 0.05$ was considered to indicate a statistically significant difference.

\section{Results}

Establishment of an asthma model. A mouse model of asthma was established as aforementioned (Fig. 1A). Crude HDM challenge induced AHR (Fig. 1B), and increased titers of HDM-specific IgE and IgG1 (Fig. 1C). In addition, recruitment of inflammatory cells to the lungs was observed in response to HDM challenge, with dense bronchial cell infiltrates (Fig. 1D). 
Table I. Primers used in reverse transcription-quantitative PCR analysis.

Primer

mmu_circ_0000909

mmu_circ_0000629

mmu_circ_0000455

mmu_circ_0001454

mmu_circ_0000723

mmu_circ_0001389

mmu_IL4

mmu_IL5

mmu_IL13

mmu_GAPDH
Sequence (5' to $\left.3^{\prime}\right)$

Forward, GCTGAGGAAGTGGTGGATGA

Reverse, TGTGATGAGTACGCGCATGT

Forward, TCTGTGGCAGGCTCTATGGC

Reverse, GCTTTGCTCCCGAAACGACT

Forward, TAAGAAAATGCCTCGCACGA

Reverse, GCAGGTTACAAAAGGCTGGC

Forward, GGCAACCACCGAAGCAGTAA

Reverse, CAAGGCAGAGAGGTGGCAGA

Forward, CACCTCGAGTCACTGTCGCA

Reverse, TCCCGGTTAATTCAGGAGCC

Forward, GGCATTGCAGAGGACCTCAA

Reverse, AGGAGGACGCACTGCTTGTG

Forward, GGTCTCAACCCCCAGCTAGT

Reverse, GCCGATGATCTCTCTCAAGTGAT

Forward, CTCTGTTGACAAGCAATGAGACG

Reverse, TCTTCAGTATGTCTAGCCCCTG

Forward, CCTGGCTCTTGCTTGCCTT

Reverse, GGTCTTGTGTGATGTTGCTCA

Forward, AGGTCGGTGTGAACGGATTTG

Reverse, TGTAGACCATGTAGTTGAGGTCA

circ, circular RNA.

Total and differential cell counts of BALF samples indicated an increased number of total inflammatory cells, with significant eosinophil recruitment to the lungs compared with that in the normal group (Fig. 1E). Lung tissue exposed to HDM also exhibited higher mRNA expression levels of IL-4, IL5 and IL-13 (Fig. 1F). These results indicated that HDM induced evident Th2 type pulmonary inflammation.

circRNA expression profile. As aforementioned, circRNA sequencing libraries were constructed and sequenced. The total number of circRNAs annotated or unannotated in circBase identified in each sample is shown in Fig. 2A. The circRNA types (exonic, intronic and intergenic) of each sample are shown in Fig. 2B, and the types of circRNAs at each chromosome position in the two groups are shown in Fig. 2C. In total, 10,924 circRNAs were identified in the control group, whereas 9,412 circRNAs were detected in the asthma model group, among which 6,402 circRNAs were expressed in both groups (Fig. 2E). The box plot in Fig. $2 \mathrm{~F}$ shows the distribution and dispersion of circRNA expression levels in each sample. Furthermore, the number of circRNAs distributed on each chromosome was examined and it was observed that the majority of circRNAs $(82 \%)$ were derived from exons (Fig. 2D).

Analysis and validation of differentially expressed circRNAs. A total of 282 circRNAs that were significantly differentially expressed in the lung tissue between the control and asthma model groups were identified, among which 152 were upregulated (red spots) and 130 were downregulated (blue spots), as shown in the heatmap (Fig. 3A) and the volcano plot (Fig. 3B). To verify sequencing expression data, six differentially expressed circRNAs (according to the results of miRanda and the fold change of circRNAs expression), including three upregulated (circ_0000909, circ_0000629 and circ_0000455) and three downregulated (circ_0001454, circ_0000723 and circ_0001389) circRNAs were selected for RT-qPCR validation (Fig. 4A). The results revealed that the relative expression levels of circ_0000629 and circ_0000455 in the asthma group were significantly increased compared with those in the control group, whereas the expression levels of circ_0000454 and circ_0000723 were significantly decreased (Fig. 4B). These results confirmed that the four circRNAs were differentially expressed.

GO and KEGG pathway analyses of differentially expressed circRNAs. GO functional classification and enrichment analysis was conducted on genes derived from circRNAs. Deeper classification and enrichment analysis of the three major functions, namely biological process, cellular component and molecular function, was performed. The results of GO functional classification were shown in Fig. 5A. GO was a classification system of function of gene, whereas DAG was a graphical display of GO enrichment data. The DAG of biological processed was shown in Fig. S1A. The DAGs of cellular components and molecular functions were shown in 
A

Sacrifice

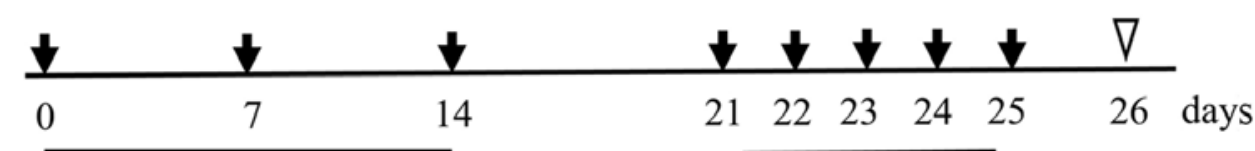

HDM $20 \mu \mathrm{g}$ s.c.

B

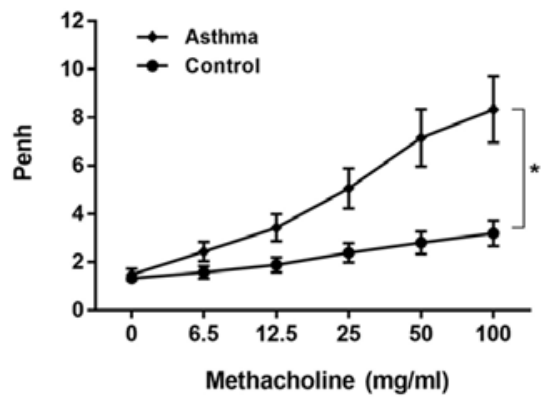

D

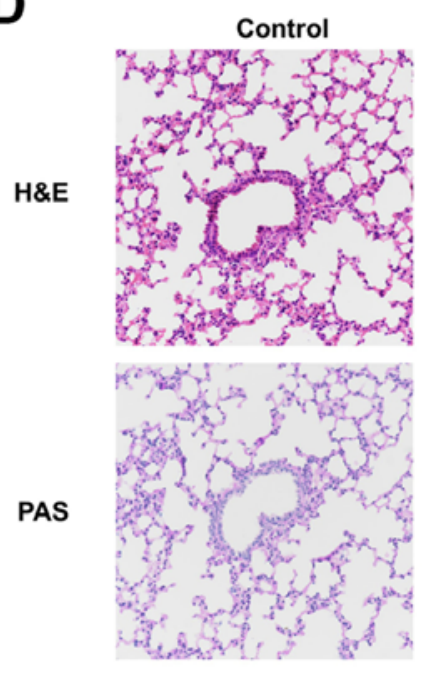

HDM $20 \mu \mathrm{g}$ Nasal drip
C

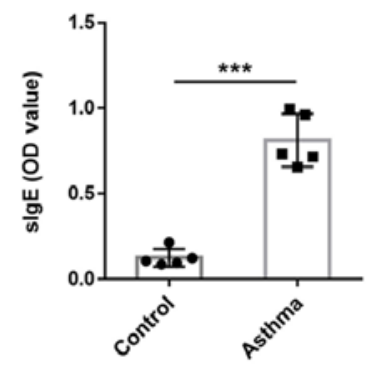

E

Control
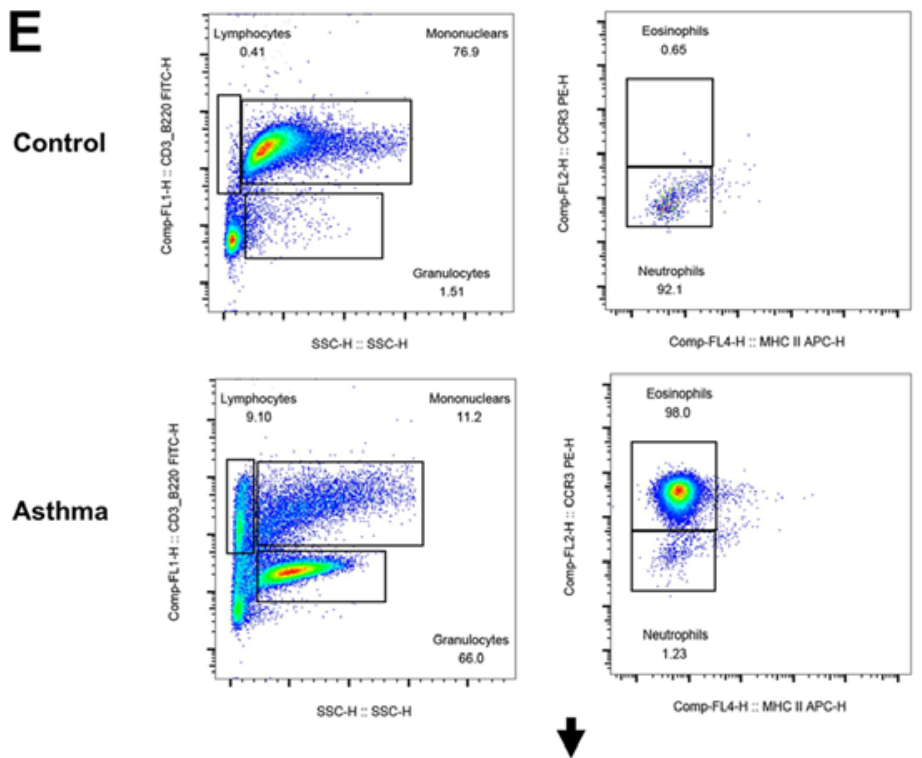
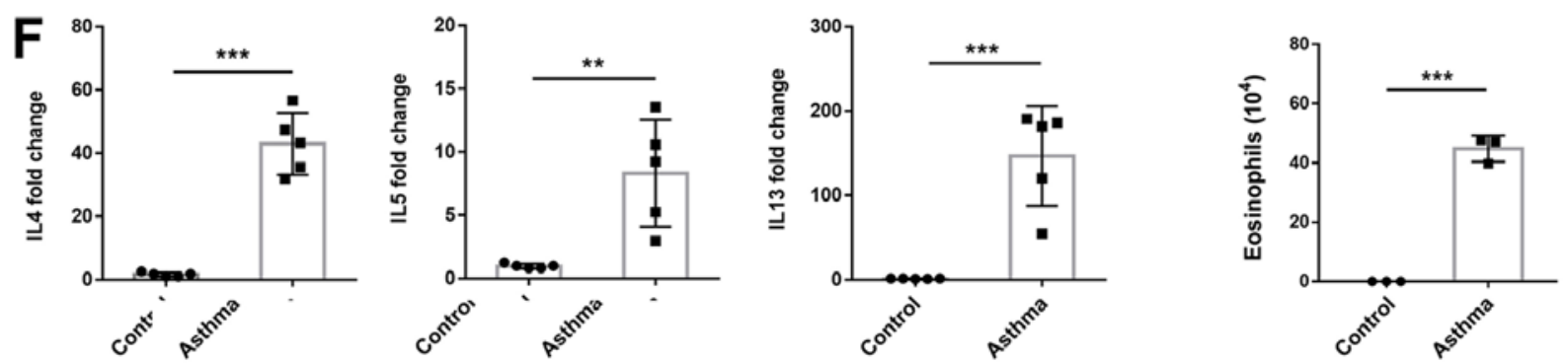

Figure 1. Establishment of a mouse model of HDM allergen-induced asthma. (A) Protocol for the generation of a murine asthma model. (B) AHR to inhaled methacholine $(6.25-100 \mathrm{mg} / \mathrm{ml}$ ). (C) ELISA of HDM-specific IgE and IgG1 in mouse serum. (D) Lung tissue histopathology (magnification, x200). Paraffin-embedded tissue sections of the lung tissue were stained with H\&E (left panel) and PAS (right panel). (E) Gating strategies for the flow cytometric analysis of BALF cells of mice challenged with PBS (control, upper panel) and HDM (asthma, lower panel). Quantitation of BALF eosinophils counts as indicated by lower panel histogram. (F) Reverse transcription-quantitative PCR for mRNA expression levels of Th2 cytokines. Data are presented as mean \pm SEM ( $n=3-5 /$ group). ${ }^{*} \mathrm{P}<0.05,{ }^{* *} \mathrm{P}<0.01,{ }^{* * * *} \mathrm{P}<0.001$. HDM, house dust mite; AHR, airway hyperresponsiveness; H\&E, hematoxylin and eosin; PAS, periodic acid-Schiff; BALF, bronchoalveolar lavage fluid.

Fig. S1B and C. The results of KEGG pathway enrichment analysis were shown in Fig. S1D demonstrating all the functions, and the results of some specific pathway enrichments were shown in Fig. 5B. The enriched GO terms were mainly associated with 'cellular process', 'biological regulation', 'metabolic process', 'regulation of biological process', and 'cellular component organization or biogenesis' (Fig. 5A). Genes associated with differentially expressed circRNAs 
A

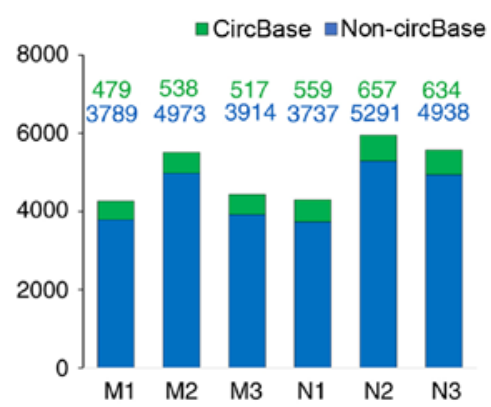

\section{C}

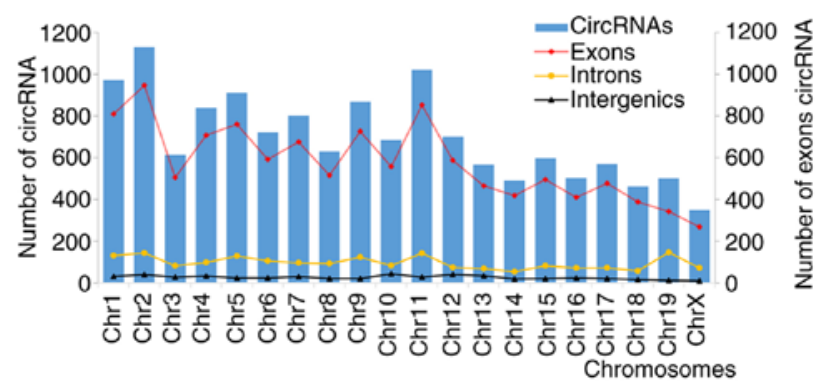

B

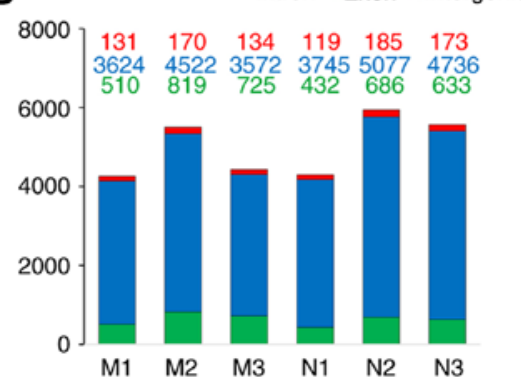

E

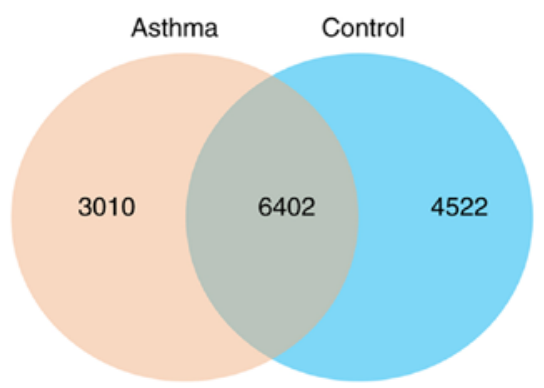

$\mathbf{F}$

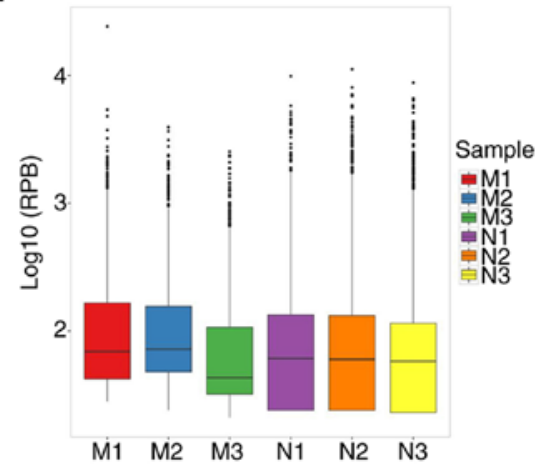

Figure 2. Abundance and characteristics of circRNAs in mouse lung tissue. (A) Annotated and non-annotated histograms of circRNA numbers in circBase. N1, N2 and N3, control mice; M1, M2 and M3, asthmatic mice. Different colors indicate different types of circRNAs, and the numbers represent the number of circRNAs. (B) Histogram of circRNA types. (C) Distribution of the types of circRNAs according to chromosome position. (D) Percentage of circRNA types. (E) Venn diagram of circRNAs. (F) Box plot of circRNA expression. The samples are shown in different colors. The abundance of circRNAs (ranging from maximum, upper quartile, median, lower quartile to minimum) is shown from top to bottom. circRNA, circularRNA.

A

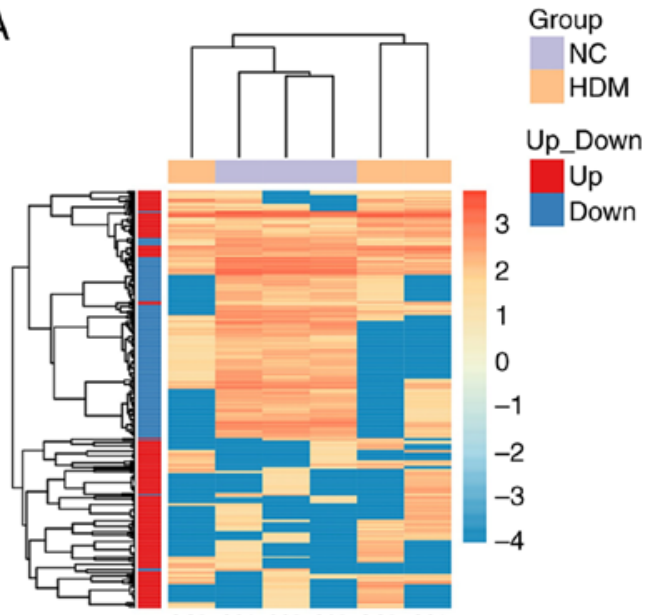

M3 N1 N2 N3 M2 N1

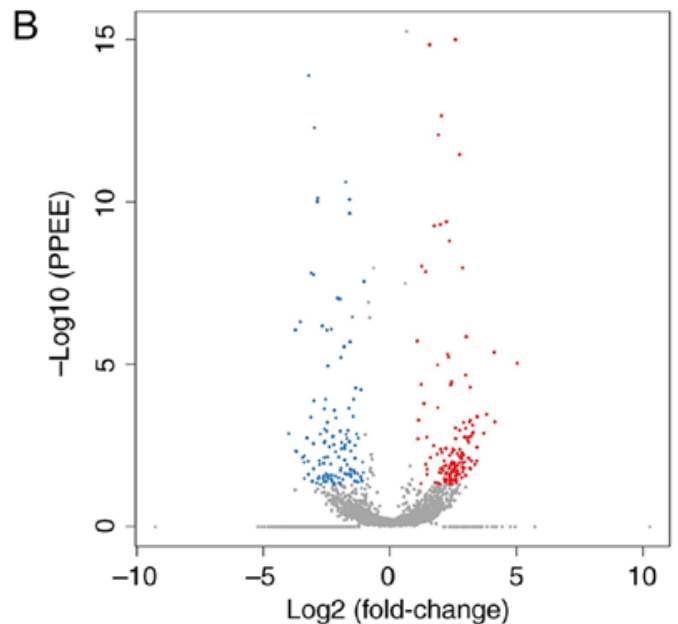

Figure 3. Analysis and validation of differentially expressed circRNAs. (A) Heat map and (B) volcano plot of the circRNAs differentially expressed between the control and asthma model groups. Blue, decreased expression; red, increased expression $(\mathrm{P}<0.05)$. circRNA, circular RNA. 
A

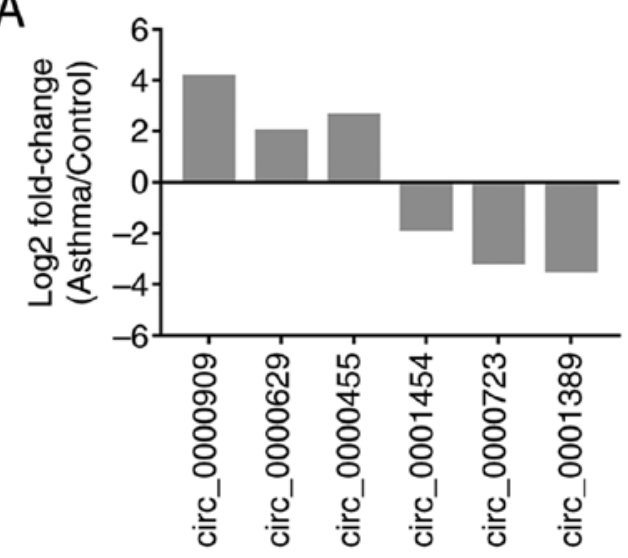

B

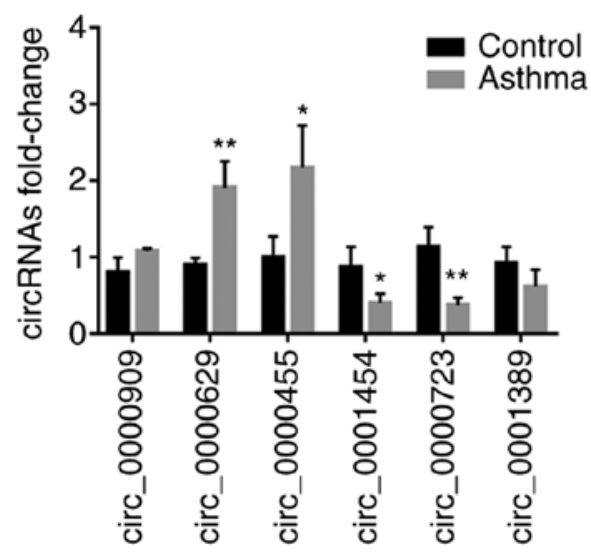

Figure 4. Validation of differentially expressed circRNAs. (A) Expression of differentially expressed circRNAs was determined by circRNA sequencing. (B) Expression of differentially expressed circRNAs was determined using quantitative PCR ( $\mathrm{n}=3 /$ group). Data are presented as mean \pm SEM ( $=3-5 /$ group). ${ }^{*} \mathrm{P}<0.05,{ }^{* *} \mathrm{P}<0.01$. circRNA, circular RNA.

were mainly enriched in 'autoimmune disease', 'cell adhesion molecules (CAMs)', 'endocytosis' and 'lipid metabolism' pathways, among others (Figs. 5B and S1D).

circRNA-miRNA regulatory network construction. circRNAs comprise a large class of post-transcriptional regulators that have been proven to act like sponges for miRNAs, thereby indirectly regulating mRNA expression $(13,14)$. Based on the calculations of miRanda, the results of differentially expressed circRNAs in circBase combined with miRNAs in miRBase were provided. In the constructed potential circRNA-miRNA network, 491 miRNAs interacted with 63 differentially expressed circRNAs (23 upregulated and 40 downregulated) (Fig. 6). The circRNA-miRNA regulatory network indicated that two of the downregulated circRNAs (circ_0001454 and circ_0000723) targeted miR-146b and miR-214, and two of these upregulated circRNAs (circ_0000455 and circ_0000629) could target miR-29b and miR-15a.

\section{Discussion}

In order to construct a reliable animal model for circRNA profiling in allergic diseases, HDM, the most common and causative allergen source, was selected (29). Notably, the model group developed apparent hypersensitivities upon challenge with methacholine. A typical Th2 type immune response was observed, with elevated expression of type 2 cytokines, including IL-4, IL-5 and IL-13, as well as IgE and IgG1 antibody titers. Pathology analysis demonstrated that the lung tissues were extensively infiltrated by inflammatory cells, such as eosinophils, which are considered indicators of allergic inflammation (30). These findings confirmed that a murine model of HDM-induced asthma was successfully established. AHR was measured by Penh, the validity of which as an index of airflow limitation was once considered controversial. Kirschvink et al (31) argued that although Penh was able to detect changes induced by pulmonary cholinergic stimulation, it poorly detected tracheal calibre modifications in rats. However, Nakaya et al (32) demonstrated that Penh could be a useful noninvasive indicator for studying nasal hypersensitivity in murine allergic rhinitis. In addition, a recent study indicated that the non-invasive Penh system was more accurate compared with the invasive ventilated lung resistance method, as ventilation induced an additional cell influx into the airways (33). It was therefore suggested that Penh could be used to evaluate hypersensitivity in asthma models.

In the present study, the majority of circRNAs (82\%) were derived from exons of protein-coding genes, whereas the others originated from intronic or intergenic genomic regions. A total of 282 circRNAs (152 upregulated and 130 downregulated) were differentially expressed in the murine allergy model group compared with in the control group. KEGG pathway analysis revealed that the differentially expressed circRNAs were strongly associated with 'cellular process' and 'biological regulation'. The analyses indicated that circRNAs performed critical functions in asthma, and may act through endocytosis, or signaling pathways that involve cell adhesion or lipid metabolism. Cell adhesion molecules have been closely implicated in various immunological disorders, including asthma, and may serve a key role in their pathogenesis by upregulating the function of eosinophils $(30,34)$. Several clinical studies have established that macrophage phagocytosis is disrupted in asthma $(35,36)$, and this disruption is more pronounced in more severe forms of asthma (37). In addition, altered sphingolipid metabolism has been reported to be associated with asthma phenotype in patients allergic to HDMs (38).

Previous studies demonstrated that circRNAs are highly evolutionarily conserved between humans and mice $(39,40)$, and some may function as miRNA sponges. In the present study, four differentially expressed circRNAs were validated by qPCR, of which two were significantly upregulated and two were significantly downregulated. The regulatory network of circRNA and miRNA indicated that two of these upregulated circRNAs (circ_0000455 and circ_0000629) could target miR-29b and miR-15a, respectively, which were previously reported to be negatively correlated with the occurrence of allergic reactions $(41,42)$. The expression levels of inducible co-stimulator, a target gene of miR-29b, were also previously shown to be elevated in the lungs of asthmatic mice, and 
A

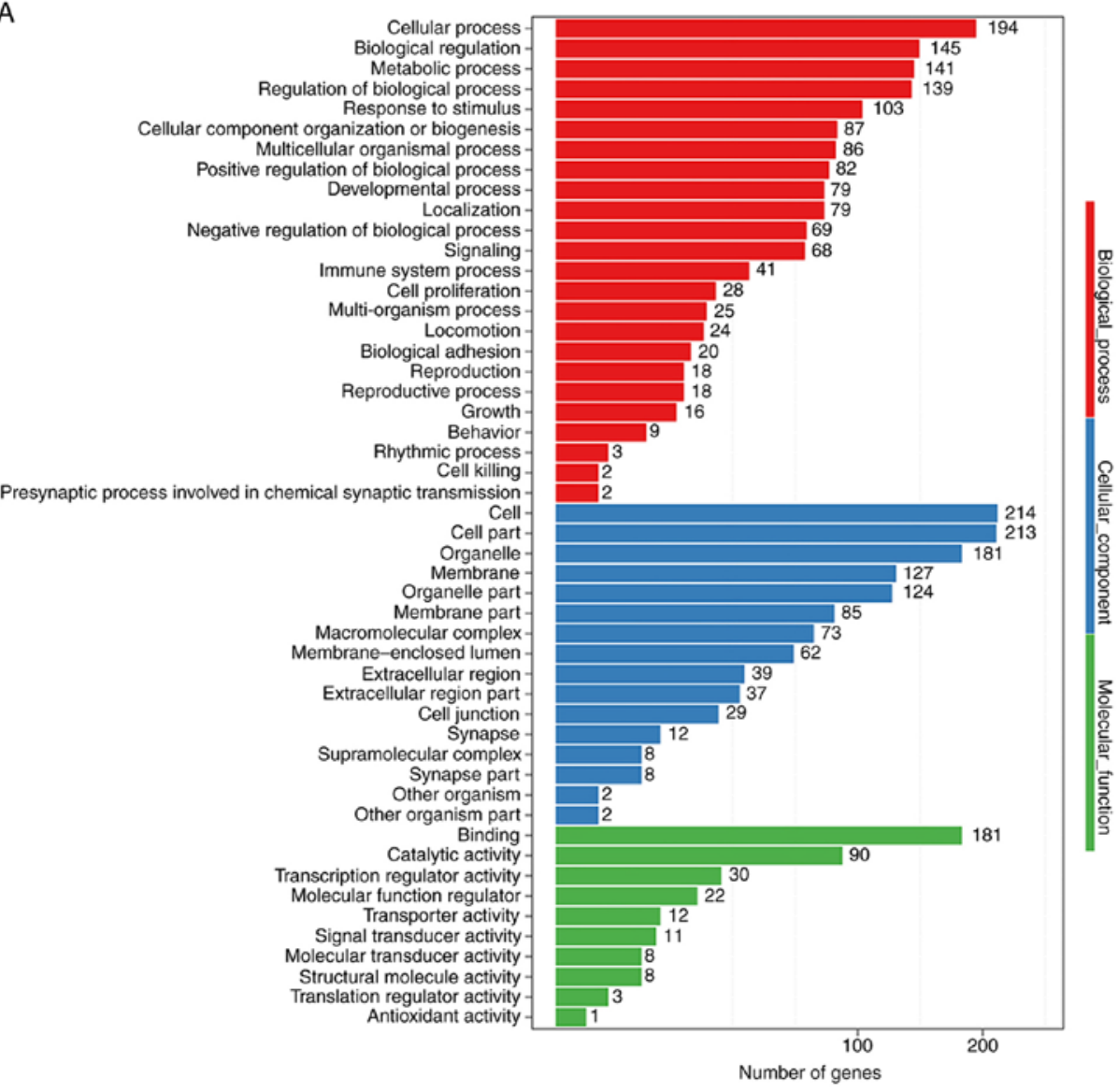

B

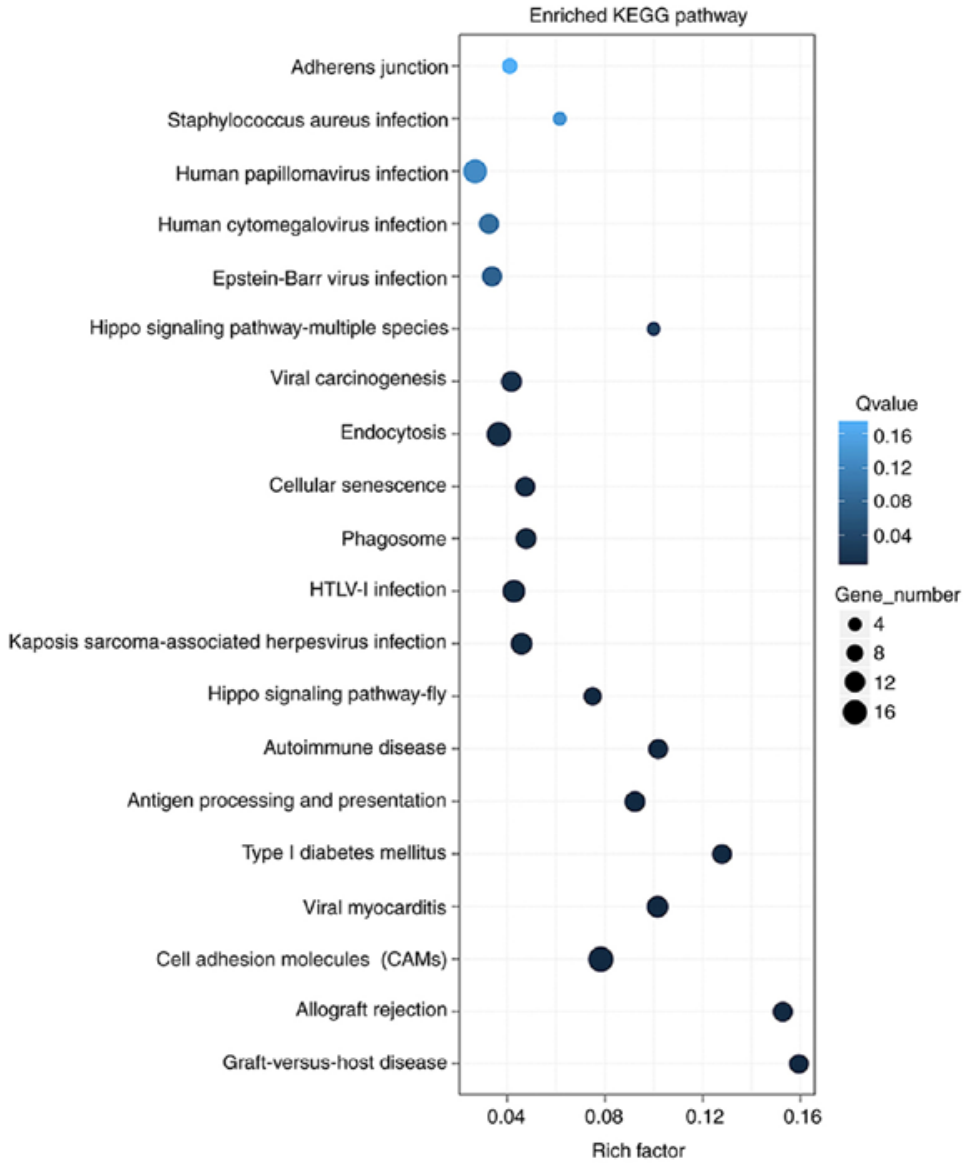

Figure 5. GO and KEGG pathway potential molecule function analyses of differentially expressed circRNAs and the regulatory network. (A) GO functional classification map of the predicted genes regulated by differentially expressed circRNAs. (B) Scatter plot of enriched KEGG pathways. GO, Gene Ontology; KEGG, Kyoto Encyclopedia of Genes and Genomes. 


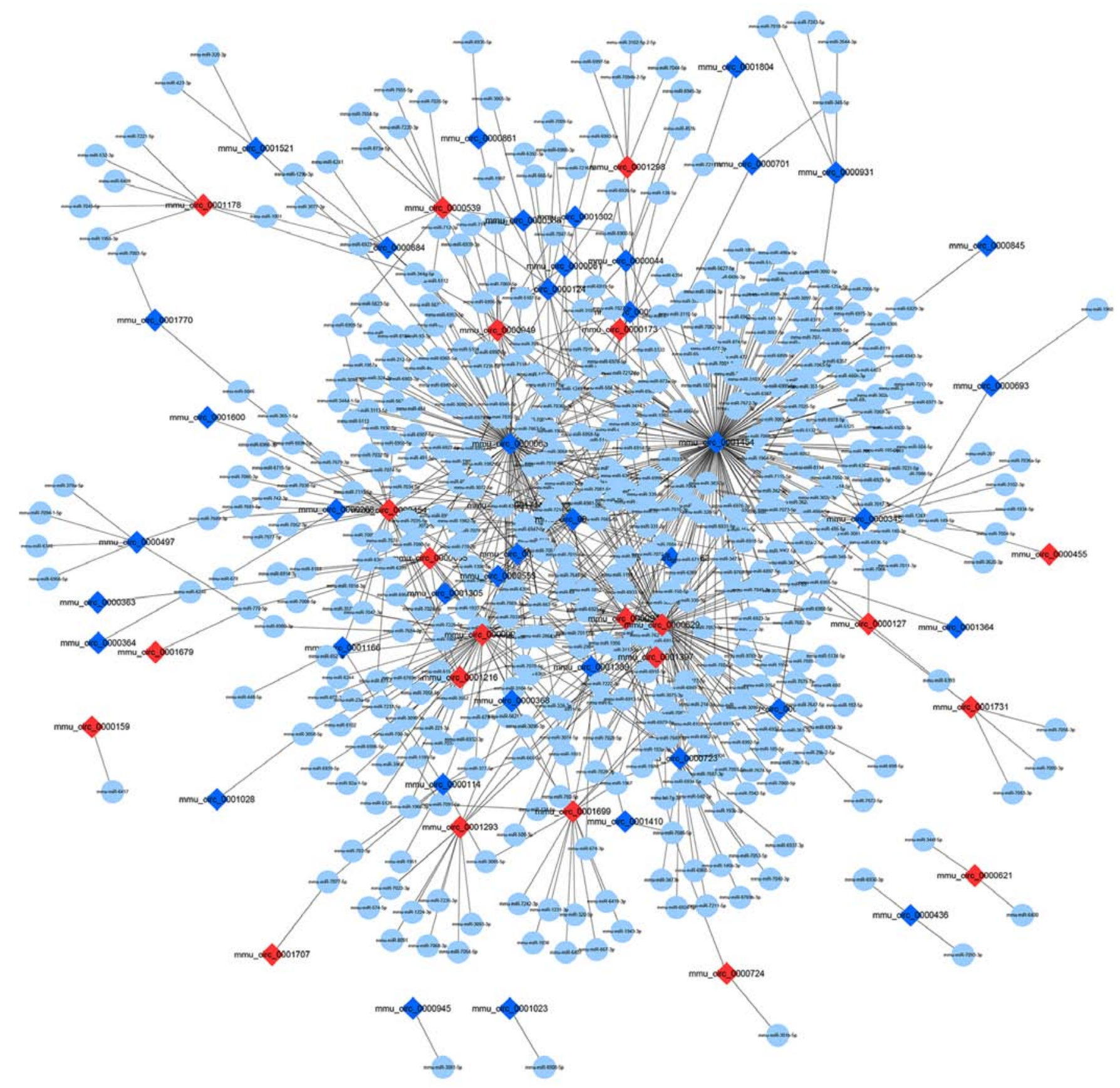

Figure 6. Regulatory network of circRNAs and miRNAs in the control and asthma model groups. Diamond nodes represent circRNAs, and round nodes represent miRNAs. Red nodes, upregulated circRNAs; blue nodes, downregulated circRNAs.

promoted Th2 cytokine production and eosinophilic inflammation (43). Furthermore, vascular endothelial growth factor, which is a target gene of mir-15a, was shown to be overexpressed in cases of Th2-mediated lung inflammation, such as asthma, and induced an asthma-like phenotype (44). By contrast, two of the downregulated circRNAs (circ_0001454 and circ_0000723) targeted miR-146b and miR-214, respectively, which were previously shown to be positively associated with asthma $(45,46)$. In a previous study, miR-146b could further propagate or help maintain the Th2 response by suppressing Th1 activation (47). Runx transcription factor, a target gene of miR-214, has been identified as a molecular link in TGF- $\beta$-induced Foxp3 expression in T regulatory cell differentiation and function, and suppressed IL-4 to affect the balance of Th1/Th2 (48). Therefore, these four circRNAs appear to be ideal circRNA candidates and future studies could be performed to investigate their association with asthma.

In conclusion, the present study identified circRNAs that were differentially expressed between the asthma model and control groups. The findings suggested that there may be a positive association between the expression of circRNAs and asthma, and provided novel insights into the etiology and pathogenesis of asthma. Future studies may employ gain- or loss-of-function assays to verify the functions of circRNAs as miRNA sponges and their involvement in the development and pathogenesis of asthma, in mice as well as in humans. 


\section{Acknowledgements}

The authors would like to thank Professor Liu (Research Center of Allergy and Immunology, Shenzhen University School of Medicine) for technical assistance, as well as his critical editing of the manuscript.

\section{Funding}

The present study was supported by the Shenzhen Fundamental Research Plan (grant no. JCYJ 20170818094217688), the Science and Technology Foundation of Guangdong Province (grant no. 2014A020212422), the National Natural Science Foundations of China (grant no. 81273275 and 31671766) and the Discipline Construction Project of Biochemistry and Molecular Biology from National Development and Reform Commission (grant no. 1452).

\section{Availability of data and materials}

The datasets used and/or analyzed during the current study are available from the corresponding author on reasonable request.

\section{Author's contributions}

HB and LX designed experiments and performed the statistical analysis. HB interpreted the data. HB, QZ, QL and MN were involved in performing the experiments. ZL, PY and $\mathrm{SC}$ aided in the experimental design. HB and LX prepared the manuscript. LX given final approval of the version to be published. LX planned and coordinated the overall research efforts. All authors read and approved the final manuscript.

\section{Ethics approval and consent to participate}

The experimental protocols of the present study were reviewed and approved by the Animal Care and Use Committee of Shenzhen University.

\section{Patient consent for publication}

Not applicable.

\section{Competing interests}

The authors declare that they have no competing interests.

\section{References}

1. Chan TF, Ji KM, Yim AK, Liu XY, Zhou JW, Li RQ, Yang KY, Li J, Li M, Law PT, et al: The draft genome, transcriptome, and microbiome of Dermatophagoides farinae reveal a broad spectrum of dust mite allergens. J Allergy Clin Immunol 135: 539-548, 2015.

2. Roberts G, Almqvist C, Boyle R, Crane J, Hogan SP, Marsland B, Saglani S and Woodfolk JA: Developments in the field of allergy in 2017 through the eyes of clinical and experimental allergy. Clin Exp Allergy 48: 1606-1621, 2018.

3. Corren J and Ziegler SF: TSLP: From allergy to cancer. Nat Immunol 20: 1603-1609, 2019.

4. Ortiz RA and Barnes KC: Genetics of allergic diseases. Immunol Allergy Clin North Am 35: 19-44, 2015.
5. Gomez JL: Epigenetics in Asthma. Curr Allergy Asthma Rep 19: $56,2019$.

6. Dai X, Zhang S and Zaleta-Rivera K: RNA: Interactions drive functionalities. Mol Biol Rep 47: 1413-1434, 2020.

7. Sonkoly E, Janson P, Majuri ML, Savinko T, Fyhrquist N, Eidsmo L, Xu N, Meisgen F, Wei T, Bradley M, et al: miR-155 is overexpressed in patients with atopic dermatitis and modulates $\mathrm{T}$-cell proliferative responses by targeting cytotoxic $\mathrm{T}$ lymphocyte-associated antigen 4. J Allergy Clin Immunol 126: 581-589. e1-e20, 2010.

8. Knolle MD, Chin SB, Rana BMJ, Englezakis A, Nakagawa R, Fallon PG, Git A and McKenzie ANJ: MicroRNA-155 protects group 2 innate lymphoid cells from apoptosis to promote type-2 immunity. Front Immunol 9: 2232, 2018.

9. Daniel E, Roff A, Hsu MH, Panganiban R, Lambert K and Ishmael F: Effects of allergic stimulation and glucocorticoids on miR-155 in CD4 ${ }^{+}$T-cells. Am J Clin Exp Immunol 7: 57-66, 2018.

10. Zhang H, Nestor CE, Zhao S, Lentini A, Bohle B, Benson M and Wang $\mathrm{H}$ : Profiling of human CD4 ${ }^{+} \mathrm{T}$-cell subsets identifies the TH2-specific noncoding RNA GATA3-AS1. J Allergy Clin Immunol 132: 1005-1008, 2013.

11. Zhang PP, Sun J and Li W: Genome-wide profiling reveals atrial fibrillation-related circular RNAs in atrial appendages. Gene 728: 144286, 2020.

12. Vidal AF, Sandoval GT, Magalhães L, Santos SE and Ribeiro-dos-Santos Â: Circular RNAs as a new field in gene regulation and their implications in translational research. Epigenomics 8: 551-562, 2016.

13. Kristensen LS, Hansen TB, Venø MT and Kjems J: Circular RNAs in cancer: Opportunities and challenges in the field. Oncogene 37: 555-565, 2018.

14. Li Y, Zheng F, Xiao X, Xie F, Tao D, Huang C, Liu D, Wang M, Wang L, Zeng F and Jiang G: CircHIPK3 sponges miR-558 to suppress heparanase expression in bladder cancer cells. EMBO Rep 18: 1646-1659, 2017.

15. Dhamija S and Menon MB: Non-coding transcript variants of protein-coding genes-what are they good for? RNA Biol 15: 1025-1031, 2018.

16. Cates EC, Fattouh R, Wattie J, Inman MD, Goncharova S, Coyle AJ, Gutierrez-Ramos JC and Jordana M: Intranasal exposure of mice to house dust mite elicits allergic airway inflammation via a GM-CSF-mediated mechanism. J Immunol 173: 6384-6392, 2004.

17. Valentine H, Williams WO and Maurer KJ: Sedation or inhalant anesthesia before euthanasia with $\mathrm{CO}_{2}$ does not reduce behavioral or physiologic signs of pain and stress in mice. J Am Assoc Lab Anim Sci 51: 50-57, 2012.

18. Van Rijt LS, Kuipers H, Vos N, Hijdra D, Hoogsteden HC and Lambrecht BN: A rapid flow cytometric method for determining the cellular composition of bronchoalveolar lavage fluid cells in mouse models of asthma. J Immunol Methods 288: 111-121, 2004.

19. Wang E, Liu X, Tu W, Do DC, Yu H, Yang L, Zhou Y, Xu D, Huang SK, Yang P, et al: Benzo(a)pyrene facilitates dermatophagoides group 1 (Der $\mathrm{f}$ 1)-induced epithelial cytokine release through aryl hydrocarbon receptor in asthma. Allergy 74: 1675-1690, 2019.

20. Gao Y, Wang J and Zhao F: CIRI: An efficient and unbiased algorithm for de novo circular RNA identification. Genome Biol 16: 4, 2015.

21. Li H and Durbin R: Fast and accurate short read alignment with Burrows-Wheeler transform. Bioinformatics 25: 1754-1760, 2009.

22. Memczak S, Jens M, Elefsinioti A, Torti F, Krueger J, Rybak A, Maier L, Mackowiak SD, Gregersen LH, Munschauer M, et al: Circular RNAs are a large class of animal RNAs with regulatory potency. Nature 495: 333-338, 2013.

23. Langmead B and Salzberg SL: Fast gapped-read alignment with Bowtie 2. Nat Methods 9: 357-359, 2012.

24. Soneson C and Delorenzi M: A comparison of methods for differential expression analysis of RNA-seq data. BMC Bioinformatics 14: 91, 2013.

25. Glažar P, Papavasileiou P and Rajewsky N: CircBase: A database for circular RNAs. RNA 20: 1666-1670, 2014.

26. Livak KJ and Schmittgen TD: Analysis of relative gene expression data using real-time quantitative PCR and the 2(-Delta Delta C(T)) method. Methods 25: 402-408, 2001.

27. Enright AJ, John B, Gaul U, Tuschl T, Sander C and Marks DS: MicroRNA targets in Drosophila. Genome Biol 5: R1, 2003. 
28. Shannon P, Markiel A, Ozier O, Baliga NS, Wang JT, Ramage D, Amin N, Schwikowski B and Ideker T: Cytoscape: A software environment for integrated models of biomolecular interaction networks. Genome Res 13: 2498-2504, 2003.

29. Miller JD: The role of dust mites in allergy. Clin Rev Allergy Immunol 57: 312-329, 2019.

30. Nakagome $\mathrm{K}$ and Nagata M: Involvement and possible role of eosinophils in asthma exacerbation. Front Immunol 9: 2220, 2018.

31. Kirschvink N, Vincke G, Onclinx C, Peck MJ and Gustin P: Comparison between pulmonary resistance and penh in anaesthetised rats with tracheal diameter reduction and after carbachol inhalation. J Pharmacol Toxicol Methods 51: 123-128, 2005.

32. Nakaya M, Dohi M, Okunishi K, Nakagome K, Tanaka R, Imamura $\mathrm{M}$, Baba S, Takeuchi N, Yamamoto $\mathrm{K}$ and Kaga $\mathrm{K}$ : Noninvasive system for evaluating allergen-induced nasal hypersensitivity in murine allergic rhinitis. Lab Invest 86: 917-926, 2006.

33. Verheijden KA, Henricks PA, Redegeld FA, Garssen J and Folkerts G: Measurement of airway function using invasive and non-invasive methods in mild and severe models for allergic airway inflammation in mice. Front Pharmacol 5: 190, 2014.

34. Kong DH, Kim YK, Kim MR, Jang JH and Lee S: Emerging roles of vascular cell adhesion molecule-1 (VCAM-1) in immunological disorders and cancer. Int J Mol Sci 19: 1057, 2018.

35. Alexis NE, Soukup J, Nierkens S and Becker S: Association between airway hyperreactivity and bronchial macrophage dysfunction in individuals with mild asthma. Am J Physiol Lung Cell Mol Physiol 280: L369-L375, 2001.

36. Fitzpatrick AM, Holguin F, Teague WG and Brown LA: Alveolar macrophage phagocytosis is impaired in children with poorly controlled asthma. J Allergy Clin Immunol 121: 1372-1378, 2008

37. Liang Z, Zhang Q, Thomas CM, Chana KK, Gibeon D, Barnes PJ, Chung KF, Bhavsar PK and Donnelly LE: Impaired macrophage phagocytosis of bacteria in severe asthma. Respir Res 15: 72 , 2014.

38. Kowal K, Żebrowska E and Chabowski A: Altered sphingolipid metabolism is associated with asthma phenotype in house dust mite-allergic patients. Allergy Asthma Immunol Res 11: 330-342, 2019.

39. Rybak-Wolf A, Stottmeister C, Glazar P, Jens M, Pino N, Giusti S, Hanan M, Behm M, Bartok O, Ashwal-Fluss R, et al: Circular RNAs in the mammalian brain are highly abundant, conserved, and dynamically expressed. Mol Cell 58: 870-885, 2015.
40. Jeck WR, Sorrentino JA, Wang K, Slevin MK, Burd CE, Liu J, Marzluff WF and Sharpless NE: Circular RNAs are abundant, conserved, and associated with ALU repeats. RNA 19: 141-157, 2013.

41. Yan J, Zhang X, Sun S, Yang T, Yang J, Wu G, Qiu Y, Yin Y and $\mathrm{Xu}$ W: miR-29b reverses $\mathrm{T}$ helper 1 cells/T helper 2 cells imbalance and alleviates airway eosinophils recruitment in OVA-induced murine asthma by targeting inducible co-stimulator. Int Arch Allergy Immunol 180: 182-194, 2019.

42. Nakano T, Inoue Y, Shimojo N, Yamaide F, Morita Y, Arima T, Tomiita $\mathrm{M}$ and Kohno Y: Lower levels of hsa-mir-15a, which decreases VEGFA, in the $\mathrm{CD}^{+} \mathrm{T}$ cells of pediatric patients with asthma. J Allergy Clin Immunol 132: 1224-1227.e12, 2013.

43. Gonzalo JA, Tian J, Delaney T, Corcoran J, Rottman JB, Lora J, Al-garawi A, Kroczek R, Gutierrez-Ramos JC and Coyle AJ: ICOS is critical for T helper cell-mediated lung mucosal inflammatory responses. Nat Immunol 2: 597-604, 2001.

44. Lee CG, Link H, Baluk P, Homer RJ, Chapoval S, Bhandari V, Kang MJ, Cohn L, Kim YK, McDonald DM and Elias JA: Vascular endothelial growth factor (VEGF) induces remodeling and enhances TH2-mediated sensitization and inflammation in the lung. Nat Med 10: 1095-1103, 2004.

45. Feng MJ, Shi F, Qiu C and Peng WK: MicroRNA-181a, -146a and $-146 \mathrm{~b}$ in spleen $\mathrm{CD}^{+} \mathrm{T}$ lymphocytes play proinflammatory roles in a murine model of asthma. Int Immunopharmacol 13: 347-353, 2012.

46. Qiu YY, Zhang YW, Qian XF and Bian T: miR-371, miR-138, miR-544, miR-145, and miR-214 could modulate Th1/Th2 balance in asthma through the combinatorial regulation of Runx3. Am J Transl Res 9: 3184-3199, 2017.

47. $\mathrm{Lu}$ TX and Rothenberg ME: Diagnostic, functional, and therapeutic roles of microRNA in allergic diseases. J Allergy Clin Immunol 132: 3-13, quiz 14.2013.

48. Lee SH, Jeong HM, Choi JM, Cho YC, Kim TS, Lee KY and Kang BY: Runx3 inhibits IL-4 production in T cells via physical interaction with NFAT. Biochem Biophys Res Commun 381: 214-217, 2009.

This work is licensed under a Creative Commons Attribution-NonCommercial-NoDerivatives 4.0 International (CC BY-NC-ND 4.0) License. 